\title{
Spectrum Properties of Hall MHD Turbulence
}

\author{
Dan HORI ${ }^{a)}$ and Hideaki MIURA \\ National Institute for Fusion Science, Toki 509-5292, Japan \\ (Received 16 November 2007 / Accepted 22 February 2008)
}

\begin{abstract}
Hall effects on small-scale turbulence are studied by means of direct numerical simulations (DNS) of both, the incompressible single-fluid equations and the incompressible Hall magnetohydrodynamics (MHD) equations. It is shown that the energy spectrum of the velocity and magnetic fields coincide with our earlier results of shell model simulations. A direct comparison between the numerical results of incompressible Hall MHD turbulence and those of incompressible single-fluid MHD turbulence reveals that the Hall term excites the small scales in the magnetic field by strengthening energy transfer from/to the scales comparable to the ion skin depth.
\end{abstract}

(c) 2008 The Japan Society of Plasma Science and Nuclear Fusion Research

Keywords: Hall MHD turbulence, Hall term, energy transfer

DOI: $10.1585 /$ pfr.3.S1053

\section{Introduction}

In recent years, the role of two-fluid effects has attracted attention in fusion plasmas [1,2], as well as astrophysical plasmas $[3,4]$. Two-fluid models, in which ion and electron flows are distinguished, are feasible to more flexible flow and magnetic configurations compared with single-fluid models [5]. Compared with a single-fluid model, additional scale lengths are introduced. For example, the Hall term in the induction equation emerges in association with the ion skin depth. The Hall term has a derivative operator of higher order compared with other nonlinear terms; therefore it is expected to stimulate energy transfer at small scales.

For studies of two-fluid effects among scales, a simpler model is more preferable so that the effects are distinguished easily from the other effects. Incompressible Hall magnetohydrodynamics (Hall MHD) provides a minimal model that expresses two-fluid effects:

$$
\begin{aligned}
& \frac{\partial \boldsymbol{u}}{\partial t}=-(\boldsymbol{u} \cdot \nabla) \boldsymbol{u}-\nabla p+\boldsymbol{j} \times \boldsymbol{B}+v \nabla^{2} \boldsymbol{u}, \\
& \frac{\partial \boldsymbol{B}}{\partial t}=\nabla \times[(\boldsymbol{u}-\varepsilon \boldsymbol{j}) \times \boldsymbol{B}]+\eta \nabla^{2} B, \\
& \nabla \cdot \boldsymbol{u}=0,
\end{aligned}
$$

where $\boldsymbol{B}$ is the magnetic field (normalized by a representative value $\left.B_{0}\right), \boldsymbol{j}(=\boldsymbol{V} \times \boldsymbol{B})$ is the current (normalized by $B_{0} / L_{0} ; L_{0}$ is the characteristic length), $\boldsymbol{u}$ is the velocity (normalized by the Alfven speed $V_{\mathrm{A}}=B_{0} / \sqrt{\mu_{0} n_{\mathrm{i}} M_{\mathrm{i}}} ; \mu_{0}$ is the permeability of vacuum, $M_{\mathrm{i}}$ is the ion mass, and $n_{\mathrm{i}}$ is the ion number density, which is assumed to be constant for simplicity), $v$ is the viscosity, and $\eta$ is the resistivity (normalized by $V_{\mathrm{A}} L_{0}$ ), and $p$ is the pressure (normalized by $\left.B_{0}^{2} / \mu_{0}\right)$. The scale parameter $\varepsilon=l_{\mathrm{i}} / L_{0}$ is called Hall parameter, where $l_{\mathrm{i}}=\sqrt{M_{\mathrm{i}} / \mu_{0} n_{\mathrm{i}} e^{2}}$ ( $e$ is an elementary

author'se-mail: hori.dan@nifs.ac.jp

${ }^{a)}$ present address: JAXA's Engineering Digital Innovation Center, 3-1-1 Yoshinodai, Sagamihara, Kanagawa 229-8510, Japan, hori.dan@jaxa.jp charge) is the ion skin depth.

An important subject with regard to Hall MHD may be the clarification of the Hall effects on energy transfer of small scales. This subject has been studied in connection with dynamo effects and the mean field theory [6,7]. However, compared with these preceding subjects on neutral turbulence (see Ref. [8] and references therein) and MHD turbulence [9], the role of Hall effects in turbulence is not fully clarified. We aim to clarify the Hall effects in turbulence for the energy transfer in the wavenumber space, by comparing directly between the MHD and Hall MHD turbulences under the same parameters and conditions. For this purpose, we first propose a new scaling theory for the energy spectra in Hall MHD turbulence taking into the contribution the Hall term to the energy flux. Next, we conduct numerical studies of Hall MHD turbulence direct numerical simulation (DNS) of both MHD and Hall MHD equations, in order to study the effects of the Hall term through direct comparison of the MHD and Hall MHD turbulences, which was not undertaken in earlier studies. In the next section, we review our previous works on Hall turbulence $[10,11]$ and propose the new scaling theory. In section 3, the MHD and Hall MHD turbulences are compared with each other by DNS. A summary is provided in the final section.

\section{Scaling and Shell Model of Hall MHD}

DNS of high-Reynolds-number turbulence requires enormous computational resources; hence, various types of reduced models have been proposed. One of these models is the Gledzer-Ohkitani-Yamada (GOY) shell model proposed by Ohkitani and Yamada [12] for neutral fluid turbulence. The essence of shell models is that governing equations are mimicked as a combination of linear terms and nonlinear coupling terms among a few Fourier modes 

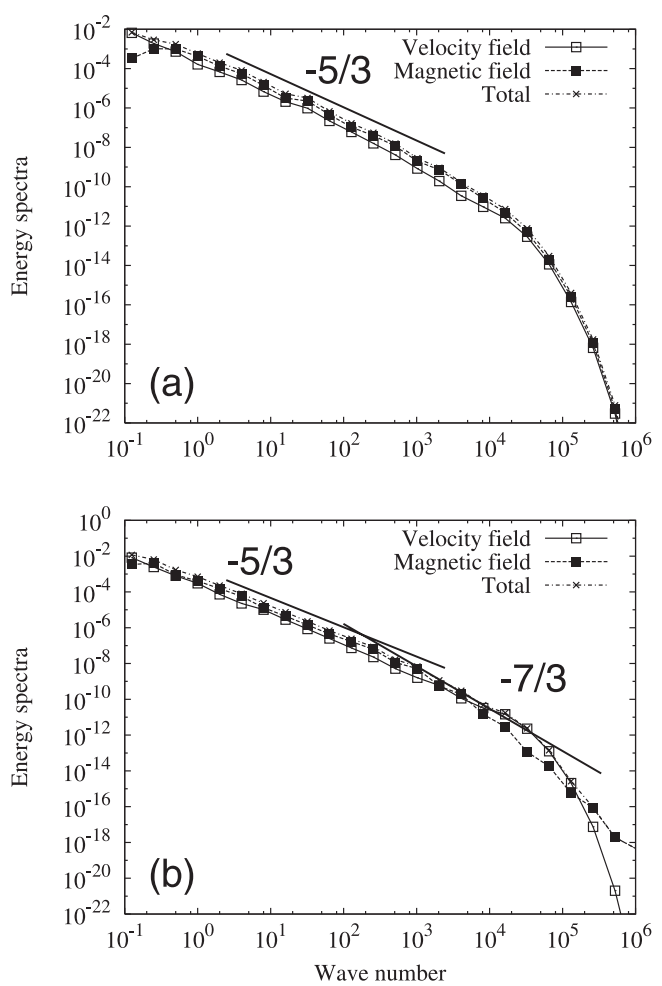

Fig. 1 Energy spectra obtained from the simulation of shell model. The MHD case is shown in the upper panel (a) and the Hall MHD case is shown in the lower panel (b).

in an artificially discretized wavenumber space. This reduction abandons the detailed dynamics of the governing equations, but enables the simulation of high-Reynoldsnumber turbulence, which cannot be performed in even the most powerful supercomputers of recent years. One of the authors (D.H.) has developed a new shell model for Hall MHD, and has performed numerical simulations in both the Hall MHD case $(\varepsilon \neq 0)$ and the single-fluid MHD case ( $\varepsilon=0)$ [10,11]. The shell model computations predict a modification of the energy spectrum by the Hall effects, suggesting modification of energy transfer from the large scales to small scales. Into aid the understanding of the next section, we review some numerical results of Hall MHD shell model reported in Refs. [10,11]. (See refer to the references for the details of the model and the scaling of the energy spectra obtained by the computations). The energy spectra for the (a) single-fluid MHD (hereafter denoted as MHD) and (b) Hall MHD (for $\varepsilon=10^{-2}$ ) are shown in Fig. 1. As observed in Fig. 1, the scaling exponents of the magnetic field are significantly modified in case of very high wavenumbers. In the case of MHD, the magnetic field spectrum is damped in the dissipation region in the same manner as the velocity energy spectrum. However, in the Hall MHD spectrum, the magnetic field spectrum indicates a $-7 / 3$ power law in the small-scale region. This contrast may reflect the contribution of the Hall term to the energy transfer in the wavenumber space. These numerical results are consistent with the following theory.
In the Kolmogorov theory (see Ref. [8] and references therein), the energy spectrum is determined from the relationships among the scales of the energy flux, viscosity, and energy spectrum, and the existence of the sub inertial range, where the contribution of the viscosity is negligible. For scaling of the energy spectrum of Hall MHD, one must take into account the contribution of the Hall term to the energy transfer. The Hall term consists of the ion skin depth and a derivative term of higher order than other nonlinear terms; therefore, the Hall term behaves as a singular perturbation and contributes to the energy transfer in the high-wavenumber region. Ion skin depth is introduced as a scale length. Here, we assume that the contribution of the Hall term is significant in the wavenumber region that is higher than the inverse of the ion skin depth $k=1 / l_{i}$, and the contribution of other nonlinear terms is dominant in the low-wavenumber region. This assumption leads to a multi-hierarchy in the energy flux:

$$
\begin{aligned}
& \text { low-wavenumber region: } \Pi^{\text {shell }}=\frac{L_{\mathrm{ch}}^{2}}{\tau_{\mathrm{ch}}^{3}}, \\
& \text { high-wavenumber region: } \Pi_{*}^{\text {shell }}=l_{\mathrm{i}} \frac{L_{\mathrm{ch}}}{\tau_{\mathrm{ch}}^{3}},
\end{aligned}
$$

where $\Pi^{\text {shell }}$ is the energy flux, $L_{\mathrm{ch}}$ is the characteristic length scale, and $\tau_{\mathrm{ch}}$ is the characteristic time scale. The energy flux function $\Pi(k)$ is typically defined by the integral of the energy transfer function $T(k)$ as

$$
\Pi(k)=\int_{k^{\prime}=k}^{\infty} T\left(k^{\prime}\right) \mathrm{d} k^{\prime},
$$

where the energy transfer function $T(k)$ originates from the nonlinear terms of the governing equations. The energy transfer function does not bring about a total increase/decrease of the energy but only contributes to modeto-mode transfer of the energy. The energy flux defined by Eq. (6) represents the energy that is transferred from wavenumbers lower than $k$ to those higher than $k$, per unit time.

Here we define the energy flux functions of the velocity and magnetic field equations for the Hall MHD equations as $\Pi_{K}$ and $\Pi_{\mathrm{M}}$, respectively, by applying the definition in Eq. (6) to Eqs. (1) and (2) as

$$
\begin{aligned}
& \Pi_{\mathrm{K}}(\boldsymbol{k})=\int_{k}^{\infty} T_{\mathrm{K}}\left(\boldsymbol{k}^{\prime}\right) \mathrm{d} \boldsymbol{k}^{\prime}, \\
& \Pi_{\mathrm{M}}(\boldsymbol{k})=\int_{k}^{\infty} T_{\mathrm{M}}\left(\boldsymbol{k}^{\prime}\right) \mathrm{d} \boldsymbol{k}^{\prime}, \\
& \Pi_{\mathrm{Tol}}(\boldsymbol{k})=\Pi_{\mathrm{K}}(\boldsymbol{k})+\Pi_{\mathrm{M}}(\boldsymbol{k}),
\end{aligned}
$$

where $T_{\mathrm{K}}\left(\boldsymbol{k}^{\prime}\right)$ is the energy transfer function of the velocity field and $T_{\mathrm{M}}\left(\boldsymbol{k}^{\prime}\right)$ is the energy transfer function of the magnetic field. $T_{\mathrm{K}}\left(\boldsymbol{k}^{\prime}\right)$ and $T_{\mathrm{M}}\left(\boldsymbol{k}^{\prime}\right)$ are defined as follows:

$$
\begin{aligned}
T_{\mathrm{K}}\left(\boldsymbol{k}^{\prime}\right)= & \mathcal{F}[\boldsymbol{u}]\left(\boldsymbol{k}^{\prime}\right)^{*} \\
& \cdot \mathcal{F}[-(\boldsymbol{u} \cdot \nabla) \boldsymbol{u}-\nabla p+\boldsymbol{j} \times \boldsymbol{B}]\left(\boldsymbol{k}^{\prime}\right), \\
T_{\mathrm{M}}\left(\boldsymbol{k}^{\prime}\right)= & \mathcal{F}[\boldsymbol{B}]\left(\boldsymbol{k}^{\prime}\right)^{*} \cdot \mathcal{F}[\nabla \times(\boldsymbol{u}-\boldsymbol{\varepsilon} \boldsymbol{j}) \times \boldsymbol{B}]\left(\boldsymbol{k}^{\prime}\right),
\end{aligned}
$$




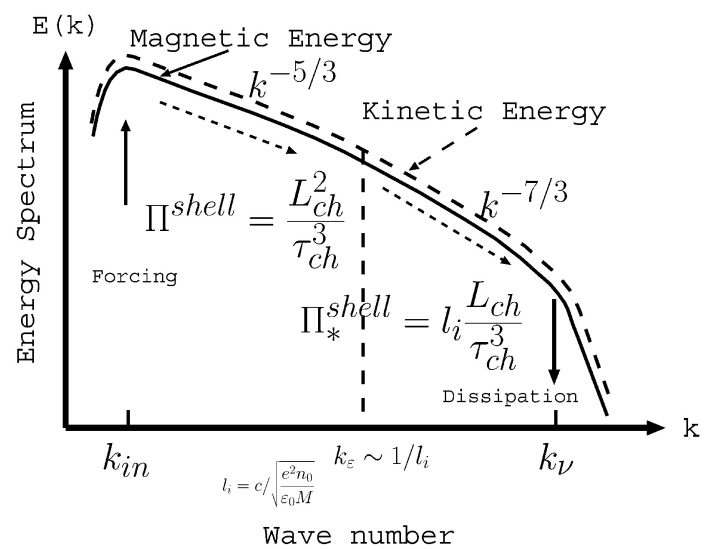

Fig. 2 Scaling of Energy spectra in Hall MHD. The contribution of Hall term to the energy transfer should be taken into account.

where $\mathcal{F}(\cdot)$ denotes the Fourier transformation and $\bullet *$ does the complex conjugate.

The energy transfer and flux functions of the shell model are also defined in the same manner as Eqs. (6)-(11). The scaling of the energy flux in the lower-wavenumber region is identical to the Kolmogorov theory. Figure 2 shows a conceptual sketch of the preceding arguments. The energy spectrum $E(k)$ in the higher-wavenumber region is scaled as $k^{-7 / 3}$, and the energy spectrum in the lowerwavenumber region is scaled as $k^{-5 / 3}$.

In the next section, we analyze the influence of the Hall term to the turbulence by DNS, while paying attention to these scaling of the energy spectra.

\section{Direct Numerical Simulation of MHD and Hall MHD Turbulences}

DNS of the decaying MHD and Hall MHD turbulences are performed for the $(2 \pi)^{3}$ triple-periodic geometry. Spatial derivatives are approximated by the pseudospectrum method and the field variables are marched into the time direction by the Runge-Kutta-Gill scheme. The aliasing error is removed by the $2 / 3$-truncation method. The number of grid points is $N^{3}=256^{3}$, so that the maximum wavenumber available in this simulation work is $k_{\max }=84$. The dissipative coefficients are $v=\eta=1 \times 10^{-2}$. For the Hall MHD turbulence, we adopt $\epsilon=0.1$, since the parameter $\epsilon=0.01$ adopted in the shell model simulation $[10,11]$ requires a huge numerical resolution. Both the MHD $(\epsilon=0)$ and Hall MHD $(\epsilon=0.1)$ turbulence simulations start from the same initial conditions in which the velocity and magnetic fields have energy spectra roughly proportional to $k^{2} \exp \left(-\left(k / k_{0}\right)^{2}\right)$ (here $k_{0}=2$ ) and random phases. No external force is imposed, so that the total energy of the velocity and the magnetic fields decays monotonically with time.

In DNS studies of fully developed turbulence, a turbulent field is often characterized by the vorticity rather than the velocity field. This is partially because the vor-
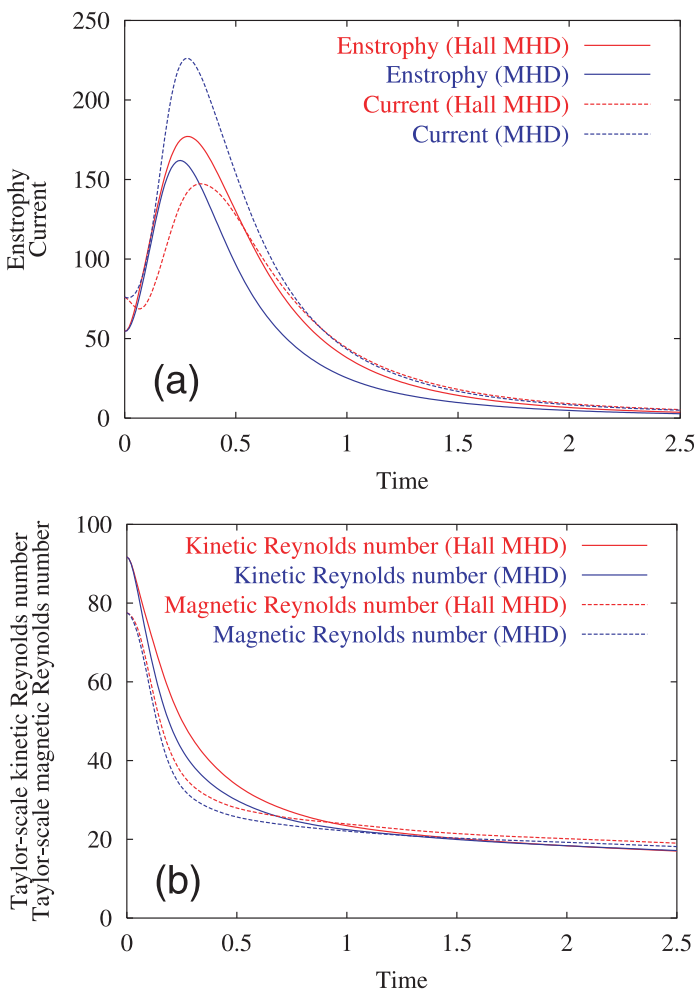

Fig. 3 Time evolutions of (a) enstrophy and current and (b) Taylor-scale Reynolds number $R e_{\lambda}$ and Taylor-scale magnetic Reynolds number $\operatorname{Re}_{\lambda}^{b}$.

ticity field $\omega$ is invariant to the Galilean transformation, and partially because the vorticity field represents smallscale structures much more than the velocity field. For the same reason, the current density field $\boldsymbol{j}$ is considered to be more suitable for studying the small-scale properties than the magnetic field. The time evolutions of the enstrophy $\left\langle|\omega|^{2} / 2\right\rangle$ and the current $\left\langle|j|^{2} / 2\right\rangle$ for the two types of turbulence are shown in Fig. 3 (a). We find in Fig. 3 (a) that the enstrophy in the Hall MHD turbulence is larger than in the MHD turbulence for the same $v$ and $\eta$. We also observe in Fig. 3 (a) that the total current is larger in the MHD turbulence than in the Hall MHD turbulence.

Another good index of fully developed turbulence is the Reynolds number $R e_{\lambda}$ based on the Taylor micro-scale [8]. Figure 3 (b) shows the time evolution of $R e_{\lambda}$ and its counterpart for the magnetic field, $R e_{\lambda}^{b}$, of the two types of turbulence. We also find that the Hall MHD turbulence has larger micro-scale Reynolds numbers $R e_{\lambda}$ and $R e_{\lambda}^{b}$ than the MHD turbulence.

The Hall effects on the scales are more directly observed by studying the energy spectra of the velocity and the magnetic field vectors. Figures 4 (a) and (b) are the plots of the energy spectra of the MHD and Hall MHD turbulences, respectively, at the peak time of the Hall MHD enstrophy in Fig. 3 (a). Since the energy is conserved as the total form $\left\langle\left(\boldsymbol{u}^{2}+\boldsymbol{B}^{2}\right)\right\rangle(\langle\cdot\rangle$ is the volume average) rather than the kinetic and magnetic energies separately in the inviscid limit, we study not only the individual spectra $u_{k}^{2}$ 

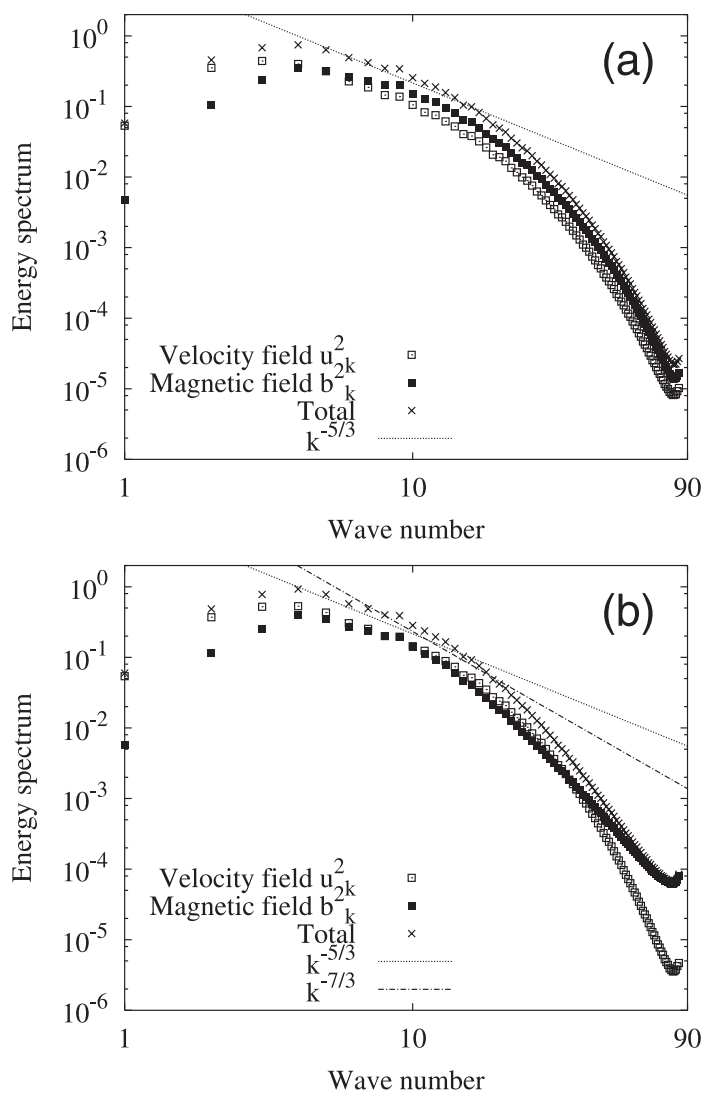

Fig. 4 Energy spectra in DNS of (a) MHD and (b) Hall MHD turbulences. The velocity and magnetic field spectra are represented by white and black boxes, respectively, at the peak time of the Hall MHD enstrophy in Fig. 3 (a). The total energy spectra $\boldsymbol{u}^{2}+\boldsymbol{B}^{2}$ is represented by the cross symbol. The dotted lines show $k^{-5 / 3}$ and the long-dashed short-dashed line shows $k^{-7 / 3}$.

and $b_{k}^{2}$ but also the total spectrum $u^{2}+b^{2}$ in these figures.

By comparing Figs. 4 (a) and (b), clear differences between the two types of turbulences are seen. In these simulations, the power law of the energy spectra is unclear due to relatively large values of $v$ and $\eta$. The $k^{-5 / 3}$ power law is barely observed around $k=10$. In the low-wavenumber region $k \leq 4$ of Fig. 4 (a), the velocity spectrum $u_{k}^{2}$ is superior to the magnetic field spectrum $b_{k}^{2}$, whereas it is opposite in the high-wavenumber region $k>4$. For $k>10$, the two spectra $u_{k}^{2}$ and $b_{k}^{2}$ have similar spectra profiles and differences can be found only in their amplitudes. On the other hand, in Fig. 4 (b), $u_{k}^{2}$ is superior to $b_{k}^{2}$ for almost all of $k \leq 30$. The magnetic field amplitude exceeds the velocity amplitude for $k>30$. In the wavenumber region $k>30$, the profile of $b_{k}^{2}$ is considerably different from that of $u_{k}^{2}$. The spectrum $b_{k}^{2}$ decays slowly as a function of the wavenumber $k$, showing that the numerical resolution of this simulation may be marginal. We find that the spectrum $b_{k}^{2}$ of the Hall MHD turbulence resembles that obtained by the shell simulation, as shown in Fig. 1 (b). These results appear to support our new scaling theories (4) and (5). Although neither $u_{k}^{2}$ nor $b_{k}^{2}$ has a sufficiently wide inertial subrange in Figs. 4 (a) and (b) because the Taylor-scale
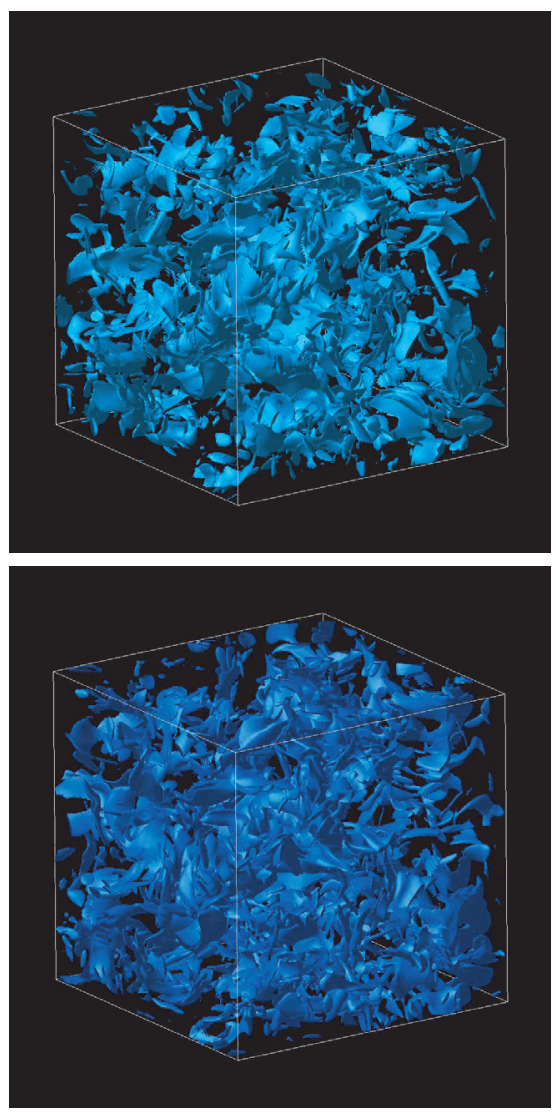

Fig. 5 Isosurfaces of the enstrophy density in (a) MHD and (b) Hall MHD turbulences.

Reynolds numbers $R e_{\lambda}$ and $R e_{\lambda}^{b}$ are not sufficiently large, the spectra in the dissipative regions of the Hall MHD turbulence in both Fig. 1 (b) and Fig. 4 (b) are slowly decaying functions of $k$. Since the magnetic energy spectra in Fig. 4 (b) appears to extend its inertial subrange due to the Hall effect, it may be expected that the $k^{-7 / 3}$-law can be observed for higher (magnetic) Reynolds number simulations. Furthermore, we find that the $u_{k}^{2}$ in $k \simeq k_{\max }$ of the Hall MHD turbulence is smaller than the $u_{k}^{2}$ in the same wavenumber region of the MHD turbulence. From these observations, it is reasonable to consider that the Hall term excites the small scales of the magnetic field and suppress the small scales of the velocity field. It supports our initial expectations that the Hall term works to excite small-scale dynamics of plasmas.

In Fig. 5, isosurfaces of the enstrophy density of the (a) MHD and (b) Hall MHD turbulences are shown. The thresholds of the isosurfaces are the same for the two figures. The volume occupied by the same level of enstrophy of Hall MHD is larger than that of MHD. The isosurfaces are more sheet-like, rather than the tubular isosurfaces seen in neutral fluid turbulence [13, 14]. In Figs. 6 (a) and (b), isosurfaces of the current density of the (a) MHD and (b) Hall MHD turbulences are shown. Again, we adopt the same thresholds in these two figures. Isosurfaces in Figs. 6(a) and (b) also have sheet-like structures. However, the volume occupied by the isosurfaces in Fig. 6 (b) 

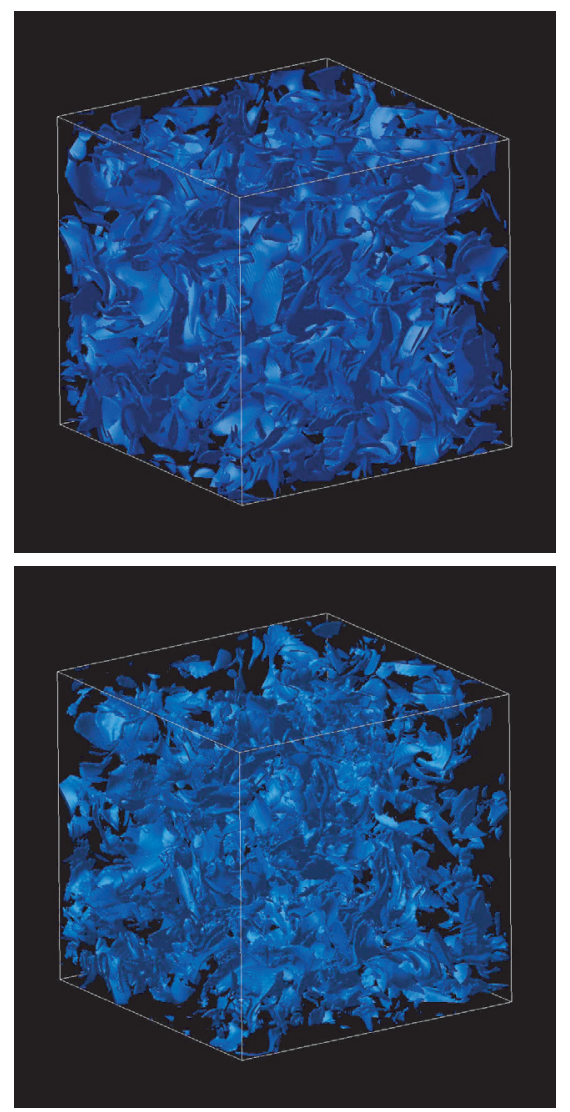

Fig. 6 Isosurfaces of the current density in (a) MHD and (b) Hall MHD turbulences.

is smaller than that in Fig. 6(a), and is consistent with the total current in Fig. 3(a). We note that the sheetlike structures in Fig. 6 should be mostly attributed to the Fourier modes of the middle wavenumber range (roughly $10 \leq k \leq 40$ ), and not those of the very large wavenumber range. The high- $k$ Fourier modes contribute to very small scales of the sheet-like structures, which have small oscillations on their surfaces. The Hall effects on each scale can be studied using the energy fluxes [8]. Because the periodic boundary condition is employed in these simulations, the energy fluxes are defined as the summations of the energy transfer functions instead of the integrals of these functions.

In Fig. 7 (a), the energy fluxes of the MHD and Hall MHD turbulences at the time of the enstrophy peak are shown. The crosses and circles correspond to $\Pi_{\mathrm{K}}$ and $\Pi_{\mathrm{M}}$, respectively. The filled boxes represent the total flux $\Pi_{\text {Tot }}$. In Fig. 7 (a), the total flux is zero or very small for small $k$. However, $\Pi_{\mathrm{K}}$ and $\Pi_{\mathrm{M}}$ are split into negative and positive values, respectively. Thus, the energy transfer between the velocity and magnetic fields is finite. The clear differences between the MHD and Hall MHD energy fluxes seen in Fig. 7 (a) should be found around $4<k<20$. The peak of $\Pi_{\text {Tot }}$ of Hall MHD is larger than that of MHD. Furthermore, $\Pi_{\mathrm{K}}$ of Hall MHD is larger than that of MHD, whereas $\Pi_{\mathrm{M}}$ of MHD is larger than that of Hall MHD. In other words, the Hall effect enhances the energy transfer to
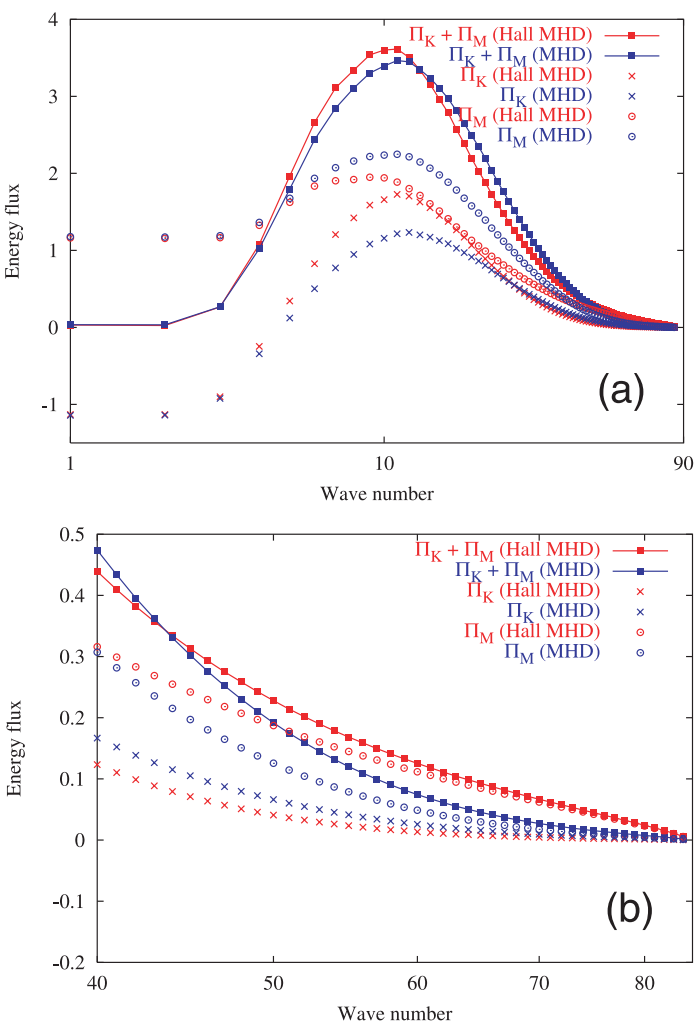

Fig. 7 (a): Energy flux in MHD and Hall MHD turbulences. (b): Magnified region of (a) for $k \geq 40$

this wavenumber region of the velocity field.

Figure 7 (b) is a magnified region of Fig. 7 (a) for $k \geq 40$. In Fig. 7 (b), while $\Pi_{\mathrm{M}}(k)$ of the MHD decays quickly as a function of $k$, that of Hall MHD decays slowly (almost as a linear function of $k$ ). Consequently, $\Pi_{\mathrm{M}}(k)$ of Hall MHD is considerably larger than that of MHD. Thus, the energy flux from low-wavenumber region to this high-wavenumber region is enhanced by the Hall term. This may explain the magnetic spectrum suppression of the Hall MHD turbulence at the moderate wavenumber region $k>10$ and the excitations of the spectrum at the high wavenumber region $k>40$ observed in Figs. 4 . The energy is transferred from the moderate-wavenumber region to the high-wavenumber region of the magnetic field. The consequence is that the magnetic field energy is weakened at the middle scales and strengthened at the small scales. In Figs. 4, we also find that the velocity spectrum in the highwavenumber region is suppressed by the Hall effect. In Fig. 7 (b), a comparison between $\Pi_{\mathrm{K}}(k)$ of Hall MHD and that of MHD shows that $\Pi_{\mathrm{K}}(k)$ is suppressed by the Hall effect in this wavenumber region, which explains the energy spectrum suppression in Hall MHD turbulence in Fig. 4.

\section{Summary}

We study the spectral properties of Hall MHD turbulence. A new scaling of the energy spectra is proposed. This scaling is consistent with the earlier numerical results. We also perform DNS of both the MHD and Hall MHD tur- 
bulences to study the role of the two-fluid effect. The energy spectra obtained by the simulations seems to be consistent with the shell model predictions for high Reynolds number and Hall parameter in the Hall MHD turbulence. These results appear to support our new scaling theory.

Our numerical results show clear difference between the MHD and Hall MHD turbulences. The analysis using the energy fluxes reveals that the Hall term is responsible for the excitations of the magnetic field at the small scales, as well as for the suppression of the velocity field at these scales. Since the Reynolds number is not very large in our numerical simulations, we need to perform simulations with a larger number of grid points. The energy flux should also be studied further, so that the energy transfer (or exchange) among various scales of the velocity and magnetic fields are clarified. In our simulations, the vorticity isosurfaces are more sheet-like structures than the tubular vortices seen in the Navier-Stokes turbulence. The Hall effects on such structure formations in turbulence is also of our interest. Such extended analysis will be performed in our future studies.

The numerical analysis was performed on the NEC SX-7 "Plasma Simulator" of National Institute for Fusion Science.
[1] K. Itoh, K. Halides et al., Phys. Plasmas 12, 062303 (2005).

[2] S.M. Mahajan and Z. Yoshida, Phys. Plasmas 7, 635 (2000).

[3] S. Balbus and C. Terquem, Astrophys. J. 552, 235 (2001).

[4] J. Shiraishi, S. Ohsaki et al., Phys. Plasmas 12, 092901 (2005).

[5] R.D. Hazeltine and J.D. Meiss, Plasma Confinement (Addison-Wesley, 1992).

[6] P. Mininni, A. Alexakis and A. Pouquet, J. Plasma Phys. 73, 377 (2007).

[7] P.D. Mininni, D.O. Gómez and S.M. Mahajan, Astrophys. J. 619, 1019 (2005).

[8] W.D. McComb, The Physics of Fluid Turbulence (Oxford University Press, 1990).

[9] D. Biskamp, Magnetohydrodynamic Turbulence (Cambridge University Press, 2003).

[10] D. Hori, M. Furukawa et al., J. Plasma Fusion Res. 81, 141 (2005).

[11] D. Hori, M. Furukawa et al., J. Plasma Phys. 72, 965 (2006).

[12] M. Yamada and K. Ohkitani, J. Phys. Soc. Jpn. 56, 4210 (1987).

[13] H. Miura and S. Kida, J. Phys. Soc. Japan 66, 1331 (1997).

[14] S. Kida and H. Miura, Europ. J. Mech. B/Fluids, 17, 471 (1998). 